Biomacromolecules. 2016 December 12; 17(12): 3964-3972. doi:10.1021/acs.biomac.6b01356.

\title{
Understanding Peptide Oligomeric State in Langmuir Monolayers of Amphiphilic 3-Helix Bundle-Forming Peptide-PEG Conjugates
}

\author{
Reidar Lund $\#+, \ddagger, \|$, JooChuan Ang ${ }^{\# \dagger}$, Jessica Y. Shu ${ }^{\dagger}$, and Ting $\mathrm{Xu}^{*}, \dagger, \ddagger, \S$ \\ tDepartment of Materials Science and Engineering, University of California, Berkeley 94720, \\ United States \\ $\S$ Department of Chemistry, University of California, Berkeley 94720, United States \\ ¥Materials Sciences Division, Lawrence Berkeley National Laboratory, Berkeley 94720, United \\ States \\ \# These authors contributed equally to this work.
}

\section{Abstract}

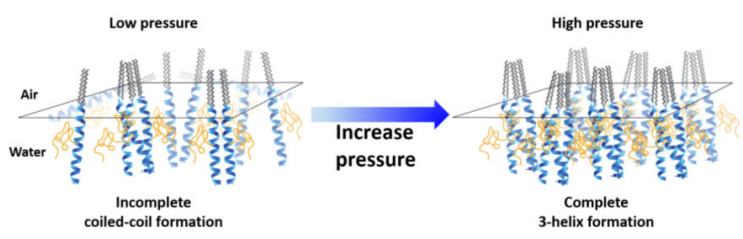

Coiled-coil peptide-polymer conjugates are an emerging class of biomaterials. Fundamental understanding of the coiled-coil oligomeric state and assembly process of these hybrid building blocks is necessary to exert control over their assembly into well-defined structures. Here, we studied the effect of peptide structure and PEGylation on the self-assembly process and oligomeric state of a Langmuir monolayer of amphiphilic coiled-coil peptide-polymer conjugates using X-ray reflectivity $(\mathrm{XR})$ and grazing-incidence X-ray diffraction (GIXD). Our results show that the oligomeric state of PEGylated amphiphiles based on 3-helix bundle-forming peptide is surface pressure dependent, a mixture of dimers and trimers was formed at intermediate surface pressure but transitions into trimers completely upon increasing surface pressure. Moreover, the interhelical distance within the coiled-coil bundle of 3-helix peptide-PEG conjugate amphiphiles was not perturbed under high surface pressure. Present studies provide valuable insights into the selfassembly process of hybrid peptide-polymer conjugates and guidance to develop biomaterials with controlled multivalency of ligand presentation.

\footnotetext{
*Corresponding Author, tingxu@ berkeley.edu. .

"Present Address

Department of Chemistry, University of Oslo, P.O. Box 1033, Blindern, N-0315 Oslo, Norway.

Supporting Information

The Supporting Information is available free of charge on the ACS Publications website at DOI: 10.1021/acs.biomac.6b01356. MALDI-TOF, circular dichroism, and 2D GIXD contour plots of the peptide-polymer conjugates (PDF).

The authors declare no competing financial interest.
} 


\section{INTRODUCTION}

The clustering of multiple copies of ligands is a common strategy in nature to overcome weak binding of individual ligand-receptor interactions ${ }^{1}$ and achieve high-affinity interfacial interactions. ${ }^{2-5}$ Natural systems routinely use the coiled-coil motif to modulate ligandreceptor interactions. ${ }^{6,7}$ The ability to control ligand presentation on surfaces, for example, density, multivalency, and interligand distance, represent new opportunities for functional control and have great potential to meet demands for tissue engineering, ${ }^{8-10}$ regenerative medicine, ${ }^{11,12}$ and drug delivery. ${ }^{13-15}$

A new class of hybrid materials based on 3-helix coiled-coil peptide-polymer conjugates was reported recently. ${ }^{16-26}$ The 3-helix peptide-polymer conjugate amphiphiles form monodisperse 3 -helix micelles (3HM) with long in vivo circulating times, ${ }^{19}$ selective accumulation within tumors over other organs in rodents, ${ }^{22}$ and are more effective than liposomes in bypassing the blood-brain barrier and accumulating within glioblastoma U87MG xenograft tumors. ${ }^{25}$ Parameters such as peptide structure, ${ }^{20}$ alkyl length, ${ }^{23}$ and position of side-conjugated $\mathrm{PEG}^{24}$ allow control over the $3 \mathrm{HM}$ kinetic stability. The coiledcoil structure presents a plausible platform for ligand presentation with well-defined multivalency on the surface of 3HM. To this end, it is important to understand the coiled-coil assembly process of peptide-polymer conjugate amphiphiles in solution and at surfaces. Our previous studies highlighted the importance of peptide structure ${ }^{20}$ and PEGylation ${ }^{24}$ on micelle kinetic stability. However, the effects of peptide structure and PEGylation on the amphiphile oligomeric state and its assembly process are unknown.

Previous studies have shown that PEGylation of 3-helix and 4-helix bundles does not adversely affect the coiled-coil formation and oligomeric state. ${ }^{16}$ Recently, small-angle Xray scattering (SAXS) of 3-helix and 4-helix peptide-polymer conjugates showed that the oligomeric state and polymer chain conformation can be determined through detailed modeling of the bundle form factor. ${ }^{18,21}$ However, it is challenging to accurately model the oligomeric state of coiled-coil amphiphiles in a micellar environment using SAXS. Coiledcoils are characterized in solution ${ }^{27-31}$ by circular-dichroism (CD) spectroscopy, sizeexclusion chromatography (SEC), and analytical ultracentrifugation (AUC), and their atomic structures can be determined by X-ray crystallography. ${ }^{32}$ Spectroscopically, CD studies of $3 \mathrm{HM}$ showed the formation of coiled-coil bundles. ${ }^{19,20}$ However, there is no direct structural information on the oligomeric state in bulk solution, especially whether if all the headgroups form coiled-coils rather than a fraction.

On Langmuir monolayers, XR and GIXD provide out-of-plane structure ${ }^{21,22}$ and in-plane spatial correlations ${ }^{33,34}$ of lateral molecular organization of amphiphiles, respectively. The orientation and packing of amphiphiles is usually a function of surface compression. ${ }^{35} \mathrm{At}$ different compressions, the out-of-plane structures would reflect various structural orientations and phase transitions of the amphiphiles at the interface. ${ }^{36,37}$ The in-plane structure would reflect the lateral packing and correlations between ordered domains, ${ }^{38-41}$ if any. The out-of plane structure and oligomeric state of amphiphilic 4-helix bundles ${ }^{42-44}$ and palmitoylated 4-helix bundles ${ }^{45}$ have previously been studied at the air-water interface using XR and GIXD. However, the coiled-coil formation process and peptide oligomeric state 
have yet to be studied for hybrid 3-helix peptide-polymer conjugate amphiphiles. To simplify the micelle formation problem in solution (a 3-D process), we choose to investigate the oligomeric state of coiled-coil formation of the 3-helix peptide-polymer conjugate amphiphiles at the air-water (2-D) surface.

Detailed structural studies confirmed that the PEGylated helical amphiphiles form a mixture of dimers and trimers at intermediate surface pressures. Upon increasing surface pressure, peptides in the amphiphile headgroups form trimers completely. This is in stark contrast to the amphiphile with a scrambled peptide sequence that does not form any higher oligomeric states under all surface pressures studied. The present study indicates the possibilities to engineer surfaces with different coiled-coil oligomeric states and opens up a viable platform to present multiple copies of ligands such as small molecules or short peptides on sub- $20 \mathrm{~nm}$ nanoparticles in a precise and controlled manner. The structural complexity coupled with the specificity of peptide-based systems, opens up new possibilities to tailor the stability of micelles and target specific therapeutic destinations within the body.

\section{EXPERIMENTAL SECTION}

\section{Synthesis and Purification}

The amphiphiles were synthesized using synthetic routes described previously. ${ }^{20}$ Briefly, 1CW (EVEALEKKVAALESKVQALEKKVEALEHGW) and SC (EGKAGEKAGAALKSGVQELEKGAEAGEGGW) peptides were synthesized using solid phase peptide synthesis. The N-terminus of the peptides were modified with a 6-(Fmocamino)hexanoic acid linker and the alkyl tails were conjugated to the $\mathrm{N}$-terminus of the peptide through reaction of palmitic acid (C16) with deprotected Fmoc-Lys(Fmoc)-OH to generate a branched alkyl tail. Cleavage of the amphiphile was achieved using a cocktail of 90:5:5 TFA/TIS/ $\mathrm{H}_{2} \mathrm{O}$ for $3 \mathrm{~h}$. The crude peptide was precipitated and washed in cold ether, isolated, and dried in vacuo. For amphiphiles with side-conjugated PEG2k, S14C mutations were performed to facilitate the site-specific coupling of maleimide-functionalized polyethylene glycol (PEG) of molecular weight $2000 \mathrm{~g} / \mathrm{mol}$ to the middle of the peptide sequences (Scheme 1). The resulting amphiphiles were purified using HPLC (Beckman Coulter) on a $\mathrm{C} 4$ column (Vydax) using a gradient of water/acetonitrile (A:B) in the presence of $0.1 \%$ TFA from $30 \% \mathrm{~B}$ to $100 \% \mathrm{~B}$ in $30 \mathrm{~min}$ at a flow rate of $8 \mathrm{~mL} / \mathrm{min}$. The purified non-PEGylated amphiphile fraction was collected at $\sim 90 \% \mathrm{~B}$, whereas fractions of PEGylated amphiphiles were collected at $\sim 85 \% \mathrm{~B}$.

\section{Langmuir Monolayers}

Isotherms were collected using a KSV 2000 Langmuir trough (Biolin Scientific) on a vibration isolation table. Typically, a $\sim 100 \mu \mathrm{M}$ solution of amphiphile dissolved in methanol was used to spread onto a phosphate buffer $(25 \mathrm{mM}, \mathrm{pH} 7.4)$ subphase. To promote the formation of a monolayer, a microliter glass syringe (Hamilton) was used to deposit the solution dropwise at the thin film of water provided by the meniscus that forms over a glass capillary penetrating the air-water interface at an oblique angle. The monolayer was allowed to equilibrate for $10 \mathrm{~min}$ before compression begins at a rate of $10 \mathrm{~mm} / \mathrm{min}$. The surface 
pressure defined as $\Pi=\gamma_{0}-\gamma$ was measured as a function of surface area assuming a monolayer and was monitored using a platinum Wilhelmy plate.

\section{Liquid Surface XR and GIXD}

XR and GIXD were performed at the 15ID-C Liquid Surface Scattering Beamline/ ChemMatCARS ${ }^{46}$ of the Advanced Photon Source/Argonne National Laboratory (APS/ ANL) using monochromatic $10 \mathrm{keV} \mathrm{X-rays.} \mathrm{For} \mathrm{XR} \mathrm{measurements,} \mathrm{the} \mathrm{spectrometer} \mathrm{was}$ kept in the reflectivity condition, where $\delta=\beta$, to collect the photons scattered with momentum transfer perpendicular to the liquid surface $\left(\mathrm{Q}_{Z}=4 \pi \sin (\delta) / \lambda\right)$. The high brilliance of the X-ray beam at APS provides high-quality XR data from 0.018 to $0.6 \AA^{-1}$. For GIXD measurements, the incident angle was set such that $Q_{Z}=0.016 \AA^{-1}$, below the critical angle of water $\left(Q_{z}=0.0217 \AA^{-1}\right)$. GIXD was scanned in the region $3.5^{\circ}<2 \theta_{x y}<$ $11.1^{\circ}$ which corresponds to $0.31<Q_{x y}<0.98 \AA^{-1}$. After deposition of the monolayer as described above, the monolayer was allowed to equilibrate for $10 \mathrm{~min}$. Compression was carried out at $10 \mathrm{~mm} / \mathrm{min}$ until the desired target surface pressure was achieved, then constant pressure was maintained during the XR measurements. The trough was translated 1 $\mathrm{mm}$ transverse to the beam to expose a fresh portion of the sample to the beam after each reflectivity scan and thereby avoided any potential radiation damage to the monolayer.

\section{XR Data Reduction and Analysis}

The reflectivity is calculated by subtracting the measured background intensity from the specular beam intensity, and then normalizing to the incident beam intensity measured by a detector placed before the sample. The fits were performed with a self-consistent approach using the program "Stochfit". ${ }^{47}$ First, the reflectivity data were analyzed using a model independent approach where layers were subdivided into typically 40 sublayers and a (nonunique) solution was found by using a stochastic search algorithm. The advantage of the program is that only the overall thickness needs to be assumed. However, the disadvantage is that the solution is likely to give unphysical oscillations. Thus, the obtained density profile was only used as a guide for further modeling. Next, this density profile was fitted using a simpler box model with a minimal number of layers (two or in a few cases, three). Subsequently, the obtained density profile was used to calculate the reflectivity curve and compared to the experimental data. Finally, refinements to the fitted curves were made by a slight variation of the parameters until a satisfactory agreement was found.

In most cases, the data could be described reasonably well using two layers by which five parameters are involved: thickness of each layer, $t_{i}$, a Gaussian width describing the roughness of the air-water surface, $\sigma_{\mathrm{a}-\mathrm{w}}$, between the layers $\sigma_{j}$, and the layer-subphase interfacial width, $\sigma_{\text {sub }}$. In some cases, satisfactorily fits could only be achieved by adding a third layer and thus two additional parameters were added $\left(t_{i+1}\right.$ and $\left.\sigma_{j+1}\right)$.

\section{Theoretical Modeling of GIXD Data}

\section{Analytical Form Factor of Aligned a-Helical Bundle-Forming Peptide-Polymer Conjugates-Cylinders aligned perpendicular to the air-water interface was used to} facilitate the description of the $a$-helical forming peptides. Furthermore, we assume only single scattering events. For a single cylinder assuming any orientation we have 


$$
P(Q)_{\mathrm{cyl}}=\int_{0}^{\pi / 2}\left|A(Q, \alpha)_{\mathrm{cyl}}\right|^{2} \sin \alpha \mathrm{d} \alpha
$$

where

$$
A(Q, \alpha)_{\mathrm{cyl}}=\frac{2 J_{1}(Q R \sin \alpha)}{Q R \sin \alpha} \frac{\sin (Q L \cos \alpha)}{Q L \cos \alpha}
$$

where $R$ and $L$ are the radius and length of the cylinder, $a$ is its angle to the scattering vector $Q$, and $J_{1}(x)$ is the first-order Bessel function.

For GIXD experiments, we only consider the Q-component in the plane of the surface, $Q_{x y}$. Equation 1 can be significantly simplified by assuming that the cylinder is perfectly aligned perpendicular to the interface. Thus, we set $a=\pi / 2$ and obtain

$$
P\left(Q_{x y}\right)_{\mathrm{cyl}}=\left(A_{\mathrm{cyl}}\left(Q_{x y}\right)\right)^{2}, A_{\mathrm{cyl}}\left(Q_{x y}\right)=\frac{2 J_{1}\left(Q_{x y} R\right)}{Q_{x y} R}
$$

As in our previous work, ${ }^{18}$ to describe solution small-angle scattering data, we employed a model describing the peptide-polymer side-conjugates as cylinders with polymer chains grafted to the sides, where the cylinders assemble into bundles with oligomeric states of either dimers or trimers. In this case, contributions from polymer chains, which nevertheless decay at lower $Q$, are disregarded.

Assuming that the 3-helix bundles are arranged in a trigonal (equilateral) arrangement, we obtain

$$
S(Q)_{\text {bundle }}^{(i)}= \begin{cases}\frac{1}{4} \cdot\left(2+2 J_{0}(2 Q R f)\right) & 2-\text { helix } \\ \frac{1}{9} \cdot\left(3+3 J_{0}(2 Q R f)\right) & 3-\text { helix }\end{cases}
$$

The scattering from an arbitrary mixture of 2- and 3-helix bundles, together with single helix peptide-polymer conjugates of fractions $f_{2}, f_{3}$, and $\left(1-f_{2}-f_{3}\right)$, respectively, can then be written as

$$
\begin{aligned}
I(Q)_{\text {bundles }}=C \cdot \phi \cdot P_{\text {cyl }}(Q) \cdot & {\left[\left(1-f_{2}-f_{3}\right)+2 f_{2} S(Q)_{\text {bundle }}^{(2)}\right.} \\
& \left.+3 f_{3} S(Q)_{\text {bundle }}^{(3)}\right]
\end{aligned}
$$

where $\phi$ is the total volume fraction and $f_{1}, f_{2}$, and $f_{3}$, are the fractions of 1-, 2-, and 3helices, respectively. Since the GIXD measurements were not performed on an absolute intensity scale a scaling constant, $C$, was included. 


\section{RESULTS AND DISCUSSION}

To decouple the effects of peptide primary structure and side-conjugated PEG on the coiledcoil formation process at the air-water interface, we synthesized 3 variants of peptidepolymer conjugate amphiphiles-dC16-1CW (non-PEGylated 3-helix amphiphile), dC161CW(PEG2k) (PEGylated 3-helix amphiphile), and dC16-SC(PEG2k) (PEGylated scrambled amphiphile) to study Langmuir monolayers of self-assembled structures and oligomeric states using XR and GIXD. dC16-SC(PEG2k) forms a mostly random coil structure with only $\sim 20 \%$ helicity, ${ }^{20}$ whereas the PEGylated and non-PEGylated 3-helix peptide amphiphiles form highly helical coiled-coil structures in aqueous solutions (SI, Figure 2).

The pressure-area isotherms of Langmuir monolayers of the various amphiphiles on a buffer subphase are shown in Figure 1. Consider dC16-1CW (Figure 1a), where the transitions are more pronounced, the isotherm can be divided into roughly three regions. First, at large mean molecular areas between $600-800 \AA^{2} /$ molecule, the surface pressure is essentially constant. This corresponds to a dilute amphiphile concentration at the water surface where the amphiphiles exist in a "gaseous" phase with little or no energy needed to compress the surface. The second region occurs at intermediate mean molecular areas between 150-600 $\AA^{2} /$ molecule where the surface pressure increases rather abruptly to $\sim 12 \mathrm{mN} / \mathrm{m}$ at $\sim 420 \AA^{2}$ / molecule then gradually to $\sim 20 \mathrm{mN} / \mathrm{m}$ upon further compression to $150 \AA^{2} /$ molecule. This transition is indicative of physical contact between the $a$-helices and they behave like a liquid-expanded phase. In this region, the hydrophobic face of the helical peptide is likely to face the air and the hydrophobic alkyl chains protrude the interface. The final, third region occurs upon further compression below $150 \AA^{2} /$ molecule, where the surface pressure increases steeply with decreasing area. This condensed phase is likely to reflect an increase in amphiphile alignment perpendicular to the surface. From a previous study using solution SAXS experiments combined with theoretical modeling, the dimensions of a single $1 \mathrm{CW} a$ helix was found to be reasonably well approximated by a cylinder with $R \cong 5 \AA$ in radius and a length of about $L \cong 45 \AA$. From these dimensions we can estimate the area of an amphiphile aligned perpendicularly or horizontally to the interface as $A_{\text {perp }}=\pi \times R^{2} \simeq 80$ $\AA^{2}$ and $A_{\text {hor }}=2 R \times L \simeq 450 \AA^{2}$. Comparing these values to the isotherm data for dC16$1 \mathrm{CW}$, we see the first transition occurs in the range of 400-500 $\AA^{2}$, while the steeper second transition occurs at smaller areas between $80-120 \AA^{2}$. These values correlate well with the estimated cross sections of the $a$-helical amphiphile. This supports the proposition that at low pressure most amphiphiles lie parallel to the interface and upon increasing the pressure, the amphiphiles orient normal to the interface.

The effect of PEGylation on the surface behavior of the helical amphiphile is shown in Figure 1b. First, dC16-1CW(PEG2k) undergoes the first transition at a smaller molecular area of $\sim 300 \AA^{2} /$ molecule compared to its non-PEGylated counterpart albeit reaching the same surface pressure. At the largest compression of $40 \AA^{2} /$ molecule, dC16-1CW(PEG2k) reached a surface pressure of $40 \mathrm{mN} / \mathrm{m}$, whereas $\mathrm{dC} 16-1 \mathrm{CW}$ obtained a surface pressure of $60 \mathrm{mN} / \mathrm{m}$ at the same compression. The first phase transition also occurs more gradually for the PEGylated dC16-1CW-(PEG2k), indicating that PEG occupies a significant fraction of the interface and mediates the interaction between $a$-helices. This is not surprising since 
PEG is known to have a large exclusion volume and steric hindrance due to the high hydration layer around PEG chains. ${ }^{48,49}$ The second transition at low molecular area, however, is only decreased by $\sim 10-20 \AA^{2} /$ molecule for the PEGylated amphiphile compared to the non-PEGylated amphiphile at the same surface pressure. This is indicative that upon interhelix contact, the presence of PEG does not significantly affect the horizontal to perpendicular transition of the amphiphiles.

Altering the peptide sequence from a helical coil-coil forming peptide (1CW) into a scrambled sequence peptide (SC) resulted in minimal hysteresis of the compression/ expansion cycle as shown in Figure 1c. In stark contrast, amphiphiles composed of the $a$ helical 1CW peptide (Figure 1a,b) displayed large hysteresis loops. The origin of the monolayer hysteresis loop under compression and expansion is likely due to the formation of coiled-coils between individual peptides at the air-water interface. Adsorption/desorption Langmuir monolayer isotherms of proteins have shown that conformational changes occur under adsorption conditions, resulting in hysteresis loops. ${ }^{50}$ In the case of the scrambled peptide, no coiled-coil formation occurs, resulting in an absence of hysteresis in the isotherm. The phase transitions, however, occurred at similar molecular areas as that of dC16-1CW(PEG2k). At the highest compression ratios, both PEGylated 1CW and SC amphiphiles attained the same surface pressure of $40 \mathrm{mN} / \mathrm{m}$.

XR was performed over an extended $Q_{Z}$ range up to about $0.6 \AA^{-1}$ covering about nine decades in intensity. To cover the relevant regions of the isotherm, XR data were collected at the various surface pressures, $10,20,30$, and $40 \mathrm{mN} / \mathrm{m}$. The reflectivity profiles for all samples, normalized to the Fresnel reflection for flat, homogeneous surfaces, are given in Figure 2.

All three variants of peptide-polymer conjugates at all surface pressures show an excess reflectivity compared to that of a Fresnel surface. Upon compression of the samples at the interface, fringes emerge indicating that the monolayers become more structured at the interface. Well-defined fringes appear at $20 \mathrm{mN} / \mathrm{m}$ for $\mathrm{dC} 16-1 \mathrm{CW}$. However, the two other amphiphiles with side-conjugated PEG2k only displayed ordered structures at a higher surface pressure of $30 \mathrm{mN} / \mathrm{m}$. This indicates that side-conjugation of PEG requires higher compression to self-assemble into ordered structures at the air-water interface.

The thickness of an interfacial layer can be determined by the period between fringes given by $d=\frac{2 \pi}{\Delta Q_{z}}$. Qualitatively, the decrease in fringe periodicity corresponds to an increase in the film thickness. The rather pronounced decrease in fringe periodicity at low $Q_{Z}$ and the increase in amplitude of the fringes correspond to a significant increase in the thickness of the monolayer in real space and reflects an accumulation of amphiphiles at the surface. To extract the out-of-plane structure of the amphiphiles at the air-water interface, XR data was fitted using a box slab (optical matrix) model where the interface is modeled as discrete layers. In addition, Gaussian smearing was used to account for interfacial roughness between layers. The electron density profiles along the air-water interface obtained from the fits are shown in Figure 3. 
First, the lowest pressure at $10 \mathrm{mN} / \mathrm{m}$ for all three amphiphiles indicate an accumulation of amphiphiles occupying a thickness of about $10 \AA$ at the interface. This corresponds to the diameter of a single helix indicating that the peptides are lying flat on the surface as nonbundled single helices. The alkyl chains are likely to be oriented up in the air occupying the portion of the surface with electron densities in the parts where $\rho / \rho_{0}$ is less than about 0.8 . The rather thin layer indicates that the alkyl chains are collapsed and lie in the plane of the air-water interface. At $20 \mathrm{mN} / \mathrm{m}$, the total thickness of the film increases to $\sim 50-60 \AA$. The thickness of this layer corresponds well to the length of the helices indicating that the peptides now are oriented normal to the interface. Upon increasing the pressure to $30 \mathrm{mN} / \mathrm{m}$ and subsequently $40 \mathrm{mN} / \mathrm{m}$, the electron density increased to about $\rho / \rho_{0} \approx 1.15$, indicating increased packing of peptide-polymer conjugate amphiphiles at the interface. The electron density is smaller than what was expected for a layer of pure peptides $\left(\rho / \rho_{0} \approx 1.34\right)$, suggesting that the peptides pack with a volume fraction of about 0.44 , the rest being water. It is interesting to compare this to the maximum packing fraction ${ }^{51}$ achievable with hexagonal packing of aligned cylinders of 0.9 . Hence, water still considerably swells the peptide helices at high pressure, indicating a significant hydration as well as inefficient packing due to bundle formation, imperfect parallel cylinders, and so on. This finding is in good agreement with our recent small-angle neutron scattering contrast variation study that showed $\sim 85 \%$ of $3 \mathrm{HM}$ is composed of water. ${ }^{26}$

The main difference between the PEGylated and non-PEGylated dC16-1CW amphiphiles, is the more pronounced and well-defined layer in the case of the non-PEGylated peptide. For dC16-1CW(PEG2k), the electron density decreases more gradually toward the interior (bottom) of the film, indicating more inhomogeneous packing at the interface. For the amphiphile comprised of the scrambled peptide sequence, the behavior looks rather different and the density profiles are generally much broader indicating that the peptide amphiphiles adsorbs like amphiphilic polymers due to the absence of $a$-helices and bundle formation. The layer structure is not as well-defined and the segments are more statistically distributed at the interface in a more diffuse manner resembling the monolayer structure of polymers previously observed. ${ }^{52-55}$

Reflectivity provides out-of-plane (z-direction) information on the film at the air-water interface. Grazing incidence diffraction at the air-water surface provides information on the in-plane ( $x y$-direction) structure and will therefore reveal any ordered structure formation such as bundling of the peptides to form coiled-coil structures. Figure 4 shows the GIXD spectra as a function of the in-plane wavevector momentum transfer, $Q_{x y}$. No significant $Q_{Z}$ dependence was found and thus the spectrum was integrated over a constant region (see Supporting Information for details).

Figure 4 shows the presence of well-defined Bragg peaks at $40 \mathrm{mN} / \mathrm{m}$ for both $\mathrm{dC} 16-1 \mathrm{CW}$ amphiphiles, regardless of the presence of PEG2k. However, dC16-SC(PEG2k) did not exhibit any Bragg peaks, even at the highest surface pressure studied of $40 \mathrm{mN} / \mathrm{m}$. Thus, there is no evidence of 2-D structures found for the amphiphile containing the scrambled peptide sequence. The monotonic decay of the GIXD profile for dC16-SC(PEG2k) could be described by a power-law decay in the form of $I \sim Q_{x y}{ }^{-0.6}$. Such power-law decay, $I \sim$ $Q_{x y}-a$, is expected for capillary waves. $I \sim Q_{x y}{ }^{-2}$ power-law has been found for air-water 
interfaces without additives (pure water). A smaller exponent can be potentially influenced by experimental smearing effects. In this work, we are not concerned with surface fluctuations and $I \sim Q_{x y}{ }^{-0.6}$ is used as a simple phenomenological description in order to subtract any contributions from capillary waves to the coherent signal from peptide in-plane structure. In order to further analyze the data, the $I \sim Q_{x y}{ }^{-0.6}$ background signal deduced from the dC16-SC(PEG2k) data was subtracted from those of dC16-1CW with and without PEG2k. The results are shown in Figure 5 for the two respective amphiphiles at surface pressures of 30 and $40 \mathrm{mN} / \mathrm{m}$.

GIXD data of dC16-1CW amphiphiles in Figure 5a show a well-defined peak in the range of $0.5-0.6 \AA^{-1}$ indicating well-defined lateral ordering at the interface. The data at both surface pressures of 30 and $40 \mathrm{mN} / \mathrm{m}$ can be well-described by the presence of only perpendicularly aligned 3-helices $\left(f_{3}=1\right)$ at the air-water interface. The peaks at $Q_{x y} \sim$ $0.557 \AA^{-1}$ at $30 \mathrm{mN} / \mathrm{m}$ and $Q_{x y} \sim 0.596 \AA^{-1}$ at $40 \mathrm{mN} / \mathrm{m}$ correspond to interhelical spacings of $d \sim 11.3 \AA$ and $\sim 10.5 \AA$, respectively. The value observed for $30 \mathrm{mN} / \mathrm{m}$ is in excellent agreement with the results from solution SAXS, ${ }^{18,21}$ where an interhelical distance was found to be about $11.6 \AA$. This small reduction in the interhelical distance at $40 \mathrm{mN} / \mathrm{m}$ suggests more severe compression of the helices within the 3-helix bundle in the absence of PEG.

The dotted line in Figure 5 illustrates a similar GIXD peak position for dC16-1CW(PEG2k) at both 30 and $40 \mathrm{mN} / \mathrm{m}$ to that of its non-PEGylated counterpart at $30 \mathrm{mN} / \mathrm{m}$. The interhelical distance corresponds to about $11.5 \AA$. The absence of surface pressure dependence on the interhelical distance of dC16-1CW(PEG2k) indicates that the PEG chains do act as "springs". Steric repulsion of the PEG chain mediates the interbundle interactions and prevents compacting of the helices within the coiled-coil bundle. Although the pressure dependence on the interhelical distance was not found for $\mathrm{dC} 16-1 \mathrm{CW}(\mathrm{PEG} 2 \mathrm{k})$ at 30 and $40 \mathrm{mN} / \mathrm{m}$, the shape of the GIXD profiles appears to be slightly different at the two pressures, indicating some differences in the coiled-coil structures formed at the surface under various surface compressions.

Detailed modeling of the GIXD spectra was performed to understand this behavior. A similar approach to previous solution SAXS description of coiled-coil/polymer conjugates was used. The model consisted of a collection of parallel cylinders to describe the helices and attached Gaussian chains to model the PEG chains. For simplicity, a two-state model was used: either perpendicular or parallel perfectly aligned bundles relative to the air-water interface. Contributions for cylinders or bundles lying perfectly parallel to the surface are not expected to give a coherent signal in $Q_{x y}$ as the scattering correlations along the length of the cylinder is parallel to $Q_{Z}$. Using this model, we allowed the peptide to form single helices, dimers (2-helices), or trimers (3-helices). The data in Figure 6 were fitted to mixtures of 1-, 2-, and 3-helices (eq 4).

The fit results in Figure 6a reveal that at $30 \mathrm{mN} / \mathrm{m}$ the data can be better described by a predominant fraction of 2-helix rather than 3-helix bundles. The best fits to the data indicate that $85 \%$ 2-helix rather than a 100\% 2-helix bundle composition applies to the surface oligomeric state at $30 \mathrm{mN} / \mathrm{m}$. Attempting to fit the data assuming a composition of $100 \% 3$ - 
helices returned a significantly worse fit, especially at low $Q_{x y}$. At $40 \mathrm{mN} / \mathrm{m}$, the results in Figure $6 \mathrm{~b}$ show the data are better fitted by $100 \% 3$-helix composition. This result indicates that complete coiled-coil bundle formation in the presence of conjugated PEG can be promoted under high compressions.

Klok and co-workers have found that $a$-helical coiled-coil peptides retain their structures upon PEGylation. ${ }^{29,30}$ PEGylation has been also found to adversely affect $\beta$-sheet formation from the conversion of $a$-helix within an alanine-rich peptide at elevated temperatures. ${ }^{56}$ Nanotubes were formed by PEGylation of tetraphenylalanine due to formation of antiparallel $\beta$-sheets and $\pi$ - $\pi$-stacking. ${ }^{57}$ As the PEG chain length increased, the nanotubes were observed to transition into fibrils. ${ }^{58}$ The delicate interplay between steric hindrance of the conjugated PEG and inter- and intramolecular interactions of the peptide governs the self-assembly of hybrid peptide-PEG materials. Here, at intermediate pressures $(\sim 30 \mathrm{mN} / \mathrm{m})$, un-PEGylated amphiphiles completely form 3-helix bundles whereas the PEGylated amphiphile exhibited mostly 2-helix formation. The observed differences between the dC16-1CW and its PEGylated analogue can be attributed to steric hindrance of the polymer chains within the monolayer. At higher pressures $(40 \mathrm{mN} / \mathrm{m})$, the interpeptide distance is supposed to be shorter and may contribute to complete 3-helix structure formation for both dC16-1CW and dC16-1CW(PEG2k). The non-PEGylated analog displayed slight compression of the helices within the coiled-coil bundle while the conjugated PEG chain may mediate the lateral pressure and avoid the lateral compression of helices.

\section{CONCLUSION}

In summary, we studied two important parameters, peptide structure and effect of PEGylation, in governing the oligomeric state and self-assembly process of coiled-coil peptide-polymer conjugate amphiphiles at the air-water interface. Combining the results from Langmuir isotherm, XR, and GIXD measurements, coiled-coil formation is highly dependent on peptide structure. PEGylated 3-helix amphiphiles exhibited a surface pressure dependent transition from a mixture of dimers and trimers at intermediate pressure to complete trimers at high pressure. Furthermore, the PEGylated 3-helix amphiphile was able to preserve the interhelical distance at high surface pressure whereas the non-PEGylated analog experienced a slight decrease in the interhelical distance upon high surface compression. The results shown here illustrate the role of peptide structure and PEGylation in the self-assembly process and oligomeric state of amphiphilic peptide-polymer conjugates in a Langmuir monolayer. Coiled-coil bundles are a promising strategy to generate ligands presented in a well-defined oligomeric state for high affinity, high specificity ligand-receptor applications.

\section{Supplementary Material}

Refer to Web version on PubMed Central for supplementary material.

\section{ACKNOWLEDGMENTS}

J.C.A., R.L., J.Y.S., and T.X. were supported by National Institutes of Health (Contract 5R21EB016947-02). We thank Binhua Lin and Mati Meron for technical support at beamline 15-ID-C of APS. ChemMatCARS Sector 15 is 
principally supported by the Divisions of Chemistry (CHE) and Materials Research (DMR), National Science Foundation, under grant number NSF/CHE-1346572. Use of the Advanced Photon Source, an Office of Science User Facility operated for the U.S. Department of Energy (DOE) Office of Science by Argonne National Laboratory, was supported by the U.S. DOE under Contract No. DE-AC02-06CH11357.

\section{REFERENCES}

(1). Mammen M, Choi SK, Whitesides GM. Polyvalent interactions in biological systems: Implications for design and use of multivalent ligands and inhibitors. Angew. Chem., Int. Ed. 1998; 37(20): 2754-2794.

(2). Lee YC, Lee RT. Carbohydrate-Protein Interactions: Basis of Glycobiology. Acc. Chem. Res. 1995; 28(8):321-327.

(3). Maheshwari G, Brown G, Lauffenburger DA, Wells A, Griffith LG. Cell adhesion and motility depend on nanoscale RGD clustering. J. Cell Sci. 2000; 113(10):1677-1686. [PubMed: 10769199]

(4). Lundquist JJ, Toone EJ. The cluster glycoside effect. Chem. Rev. 2002; 102(2):555-578. [PubMed: 11841254]

(5). Gestwicki JE, Cairo CW, Strong LE, Oetjen KA, Kiessling LL. Influencing receptor-ligand binding mechanisms with multivalent ligand architecture. J. Am. Chem. Soc. 2002; 124(50): 14922-14933. [PubMed: 12475334]

(6). Arnold M, Cavalcanti-Adam EA, Glass R, Blummel J, Eck W, Kantlehner M, Kessler H, Spatz JP. Activation of integrin function by nanopatterned adhesive interfaces. ChemPhysChem. 2004; 5(3):383-388. [PubMed: 15067875]

(7). Margadant C, Monsuur HN, Norman JC, Sonnenberg A. Mechanisms of integrin activation and trafficking. Curr. Opin. Cell Biol. 2011; 23(5):607-614. [PubMed: 21924601]

(8). Sontjens SHM, Nettles DL, Carnahan MA, Setton LA, Grinstaff MW. Biodendrimer-based hydrogel scaffolds for cartilage tissue repair. Biomacromolecules. 2006; 7(1):310-316. [PubMed: 16398530]

(9). Hahn MS, Miller JS, West JL. Three-Dimensional Biochemical and Biomechanical Patterning of Hydrogels for Guiding Cell Behavior. Adv. Mater. 2006; 18(20):2679-2684.

(10). Walters NJ, Gentleman E. Evolving insights in cell-matrix interactions: Elucidating how nonsoluble properties of the extracellular niche direct stem cell fate. Acta Biomater. 2015; 11:3-16. [PubMed: 25266503]

(11). Petrie TA, Raynor JE, Dumbauld DW, Lee TT, Jagtap S, Templeman KL, Collard DM, Garcia AJ. Multivalent Integrin-Specific Ligands Enhance Tissue Healing and Biomaterial Integration. Sci. Transl. Med. 2010; 2(45) 45ra6010.1126/scitranslmed.3001002.

(12). Seelbach RJ, Fransen P, Peroglio M, Pulido D, Lopez-Chicon P, Duttenhoefer F, Sauerbier S, Freiman T, Niemeyer P, Semino C, Albericio F, Alini M, Royo M, Mata A, Eglin D. Multivalent dendrimers presenting spatially controlled clusters of binding epitopes in thermoresponsive hyaluronan hydrogels. Acta Biomater. 2014; 10(10):4340-4350. [PubMed: 24993802]

(13). Poon Z, Chen S, Engler AC, Lee H.-i. Atas E, von Maltzahn G, Bhatia SN, Hammond PT. Ligand-Clustered "Patchy" Nanoparticles for Modulated Cellular Uptake and In Vivo Tumor Targeting. Angew. Chem., Int. Ed. 2010; 49(40):7266-7270.

(14). Elias DR, Poloukhtine A, Popik V, Tsourkas A. Effect of ligand density, receptor density, and nanoparticle size on cell targeting. Nanomedicine. 2013; 9(2):194-201. [PubMed: 22687896]

(15). Gray BP, Li S, Brown KC. From Phage Display to Nanoparticle Delivery: Functionalizing Liposomes with Multivalent Peptides Improves Targeting to a Cancer Biomarker. Bioconjugate Chem. 2013; 24(1):85-96.

(16). Shu JY, Tan C, DeGrado WF, Xu T. New Design of Helix Bundle Peptide-Polymer Conjugates. Biomacromolecules. 2008; 9(8):2111-2117. [PubMed: 18627200]

(17). Xu T, Shu J. Coiled-coil helix bundle, a peptide tertiary structural motif toward hybrid functional materials. Soft Matter. 2010; 6(2):212-217.

(18). Shu JY, Lund R, Xu T. Solution Structural Characterization of Coiled-Coil Peptide-Polymer SideConjugates. Biomacromolecules. 2012; 13(6):1945-1955. [PubMed: 22575010] 
(19). Dong H, Dube N, Shu JY, Seo JW, Mahakian LM, Ferrara KW, Xu T. Long-Circulating 15 nm Micelles Based on Amphiphilic 3-Helix Peptide-PEG Conjugates. ACS Nano. 2012; 6(6):53205329. [PubMed: 22545944]

(20). Dong H, Shu JY, Dube N, Ma Y, Tirrell MV, Downing KH, Xu T. 3-Helix Micelles Stabilized by Polymer Springs. J. Am. Chem. Soc. 2012; 134(28):11807-11814. [PubMed: 22731391]

(21). Lund R, Shu J, Xu T. A Small-Angle X-ray Scattering Study of $a$-helical Bundle-Forming Peptide-Polymer Conjugates in Solution: Chain Conformations. Macromolecules. 2013; 46(4): 1625-1632.

(22). Dube N, Shu JY, Dong H, Seo JW, Ingham E, Kheirolomoom A, Chen P-Y, Forsayeth J, Bankiewicz K, Ferrara KW, Xu T. Evaluation of Doxorubicin-Loaded 3-Helix Micelles as Nanocarriers. Biomacromolecules. 2013; 14(10):3697-3705. [PubMed: 24050265]

(23). Dube N, Seo JW, Dong H, Shu JY, Lund R, Mahakian LM, Ferrara KW, Xu T. Effect of Alkyl Length of Peptide-Polymer Amphiphile on Cargo Encapsulation Stability and Pharmacokinetics of 3-Helix Micelles. Biomacromolecules. 2014; 15(8):2963-2970. [PubMed: 24988250]

(24). Dong H, Lund R, Xu T. Micelle Stabilization via Entropic Repulsion: Balance of Force Directionality and Geometric Packing of Subunit. Biomacromolecules. 2015; 16(3):743-747. [PubMed: 25575164]

(25). Seo JW, Ang J, Mahakian LM, Tam S, Fite B, Ingham ES, Beyer J, Forsayeth J, Bankiewicz KS, $\mathrm{Xu}$ T, Ferrara KW. Self-assembled 20-nm 64Cu-micelles enhance accumulation in rat glioblastoma. J. Controlled Release. 2015; 220:51-60.

(26). Ang J, Ma D, Lund R, Keten S, Xu T. Internal Structure of $15 \mathrm{~nm}$ 3-Helix Micelle Revealed by Small-Angle Neutron Scattering and Coarse-Grained MD Simulation. Biomacromolecules. 2016; 17(10):3262-3267. [PubMed: 27584005]

(27). Graddis TJ, Myszka DG, Chaiken IM. Controlled formation of model homo- and heterodimer coiled coil polypeptides. Biochemistry. 1993; 32(47):12664-12671. [PubMed: 8251485]

(28). Sakamoto H, Lewis MS, Kodama H, Appella E, Sakaguchi K. Specific sequences from the carboxyl terminus of human p53 gene product form anti-parallel tetramers in solution. Proc. Natl. Acad. Sci. U. S. A. 1994; 91(19):8974-8978. [PubMed: 8090755]

(29). Vandermeulen GWM, Tziatzios C, Klok HA. Reversible self-organization of poly(ethylene glycol)-based hybrid block copolymers mediated by a De Novo four-stranded alpha-helical coiled coil motif. Macromolecules. 2003; 36(11):4107-4114.

(30). Vandermeulen GWM, Tziatzios C, Duncan R, Klok H-A. PEG-Based Hybrid Block Copolymers Containing $a$-Helical Coiled Coil Peptide Sequences: Control of Self-Assembly and Preliminary Biological Evaluation. Macromolecules. 2005; 38(3):761-769.

(31). Malik L, Nygaard J, Christensen NJ, Streicher WW, Thulstrup PW, Arleth L, Jensen KJ. Selfassembly of designed coiled coil peptides studied by small-angle X-ray scattering and analytical ultracentrifugation. J. Pept. Sci. 2013; 19(5):283-292. [PubMed: 23505212]

(32). Parry DAD, Fraser RDB, Squire JM. Fifty years of coiled-coils and alpha-helical bundles: A close relationship between sequence and structure. J. Struct. Biol. 2008; 163(3):258-269. [PubMed: 18342539]

(33). Dutta P, Peng JB, Lin B, Ketterson JB, Prakash M, Georgopoulos P, Ehrlich S. X-ray diffraction studies of organic monolayers on the surface of water. Phys. Rev. Lett. 1987; 58(21):2228-2231. [PubMed: 10034686]

(34). Kjaer K, Als-Nielsen J, Helm CA, Laxhuber LA, Mohwald H. Ordering in lipid monolayers studied by synchrotron xray diffraction and fluorescence microscopy. Phys. Rev. Lett. 1987; 58(21):2224-2227. [PubMed: 10034685]

(35). Langmuir I. The Constitution and Fundamental Properties of Solids and Liquids. II. Liquids. 1. J. Am. Chem. Soc. 1917; 39(9):1848-1906.

(36). Bosio L, Benattar JJ, Rieutord F. X-ray Reflectivity of a Langmuir Monolayer on water. Rev. Phys. Appl. 1987; 22(8):775-778.

(37). Grundy MJ, Richardson RM, Roser SJ, Penfold J, Ward RC. X-ray and neutron reflectivity from spread monolayers. Thin Solid Films. 1988; 159(1):43-52.

(38). Lin B, Shih MC, Bohanon TM, Ice GE, Dutta P. Phase diagram of a lipid monolayer on the surface of water. Phys. Rev. Lett. 1990; 65(2):191-194. [PubMed: 10042576] 
(39). Jacquemain D, Wolf SG, Leveiller F, Deutsch M, Kjaer K, Alsnielsen J, Lahav M, Leiserowitz L. 2-Dimensional Crystallography of Amphiphilic Molecules at the Air-Water Interface. Angew. Chem., Int. Ed. Engl. 1992; 31(2):130-152.

(40). Durbin MK, Malik A, Ghaskadvi R, Shih MC, Zschack P, Dutta P. X-ray Diffraction Study of a Recently Identified Phase Transition in Fatty Acid Langmuir Monolayers. J. Phys. Chem. 1994; 98(7):1753-1755.

(41). Foster WJ, Shih MC, Pershan PS. The structure of a Langmuir monolayer of methyl eicosanoate as determined by x-ray diffraction and Brewster angle microscopy. J. Chem. Phys. 1996; 105(8): 3307-3315.

(42). Ye S, Discher BM, Strzalka J, Xu T, Wu SP, Noy D, Kuzmenko I, Gog T, Therien MJ, Dutton PL, Blasie JK. Amphiphilic Four-Helix Bundle Peptides Designed for Light-Induced Electron Transfer Across a Soft Interface. Nano Lett. 2005; 5(9):1658-1667. [PubMed: 16159202]

(43). Strzalka J, Xu T, Tronin A, Wu SP, Miloradovic I, Kuzmenko I, Gog T, Therien MJ, Blasie JK. Structural Studies of Amphiphilic 4-Helix Bundle Peptides Incorporating Designed Extended Chromophores for Nonlinear Optical Biomolecular Materials. Nano Lett. 2006; 6(11):23952405. [PubMed: 17090064]

(44). Krishnan V, Tronin A, Strzalka J, Fry HC, Therien MJ, Blasie JK. Control of the Orientational Order and Nonlinear Optical Response of the 'Push-Pull' Chromophore RuPZn via Specific Incorporation into Densely Packed Monolayer Ensembles of an Amphiphilic Four-Helix Bundle Peptide: Characterization of the Peptide-Chromophore Complexes. J. Am. Chem. Soc. 2010; 132(32):11083-11092. [PubMed: 20698674]

(45). Strzalka J, Chen X, Moser CC, Dutton PL, Ocko BM, Blasie JK. X-ray Scattering Studies of Maquette Peptide Monolayers. 1. Reflectivity and Grazing Incidence Diffraction at the Air/Water Interface. Langmuir. 2000; 16(26):10404-10418.

(46). Lin B, Meron M, Gebhardt J, Graber T, Schlossman ML, Viccaro PJ. The liquid surface/interface spectrometer at Chem-MatCARS synchrotron facility at the Advanced Photon Source. Phys. B. 2003; 336(1-2):75-80.

(47). Danauskas SM, Li D, Meron M, Lin B, Lee KYC. Stochastic fitting of specular X-ray reflectivity data using StochFit. J. Appl. Crystallogr. 2008; 41:1187-1193.

(48). Atha DH, Ingham KC. Mechanism of Precipitation of Proteins by Polyethylene Glycols Analysis in terms of excluded volume. J. Biol. Chem. 1981; 256(23):2108-2117.

(49). Maisano G, Majolino D, Migliardo P, Venuto S, Aliotta F, Magazu S. Sound velocity and hydration phenomena in aqueous polymeric solutions. Mol. Phys. 1993; 78(2):421-435.

(50). Chaudhary N, Nagaraj R. Self-assembly of short amyloidogenic peptides at the air-water interface. J. Colloid Interface Sci. 2011; 360(1):139-147. [PubMed: 21550612]

(51). Trovato A, Hoang TX, Banavar JR, Maritan A. Symmetry, shape, and order. Proc. Natl. Acad. Sci. U. S. A. 2007; 104(49):19187-19192. [PubMed: 18032605]

(52). Li Z, Zhao MW, Quinn J, Rafailovich MH, Sokolov J, Lennox RB, Eisenberg A, Wu XZ, Kim MW, Sinha SK, Tolan M. X-ray reflectivity of diblock copolymer monolayers at the air/water interface. Langmuir. 1995; 11(12):4785-4792.

(53). Thomas RK, Penfold J. Neutron and X-ray reflectometry of interfacial systems in colloid and polymer chemistry. Curr. Opin. Colloid Interface Sci. 1996; 1(1):23-33.

(54). An SW, Thomas RK, Baines FL, Billingham NC, Armes SP, Penfold J. Neutron reflectivity of adsorbed water-soluble block copolymers at the air/water interface: The effects of composition and molecular weight. Macromolecules. 1998; 31(22):7877-7885.

(55). Faure MC, Bassereau P, Lee LT, Menelle A, Lheveder C. Phase transitions in monolayers of PSPEO copolymer at the air-water interface. Macromolecules. 1999; 32(25):8538-8550.

(56). Top A, Roberts CJ, Kiick KL. Conformational and Aggregation Properties of a PEGylated Alanine-Rich Polypeptide. Biomacromolecules. 2011; 12(6):2184-2192. [PubMed: 21553871]

(57). Tzokova N, Fernyhough CM, Topham PD, Sandon N, Adams DJ, Butler MF, Armes SP, Ryan AJ. Soft Hydrogels from Nanotubes of Poly(ethylene oxide)-Tetraphenylalanine Conjugates Prepared by Click Chemistry. Langmuir. 2009; 25(4):2479-2485. [PubMed: 19161273] 
(58). Tzokova N, Fernyhough CM, Butler MF, Armes SP, Ryan AJ, Topham PD, Adams DJ. The Effect of PEO Length on the Self-Assembly of Poly(ethylene oxide)-Tetrapeptide Conjugates Prepared by "Click" Chemistry. Langmuir. 2009; 25(18):11082-11089. [PubMed: 19685857] 

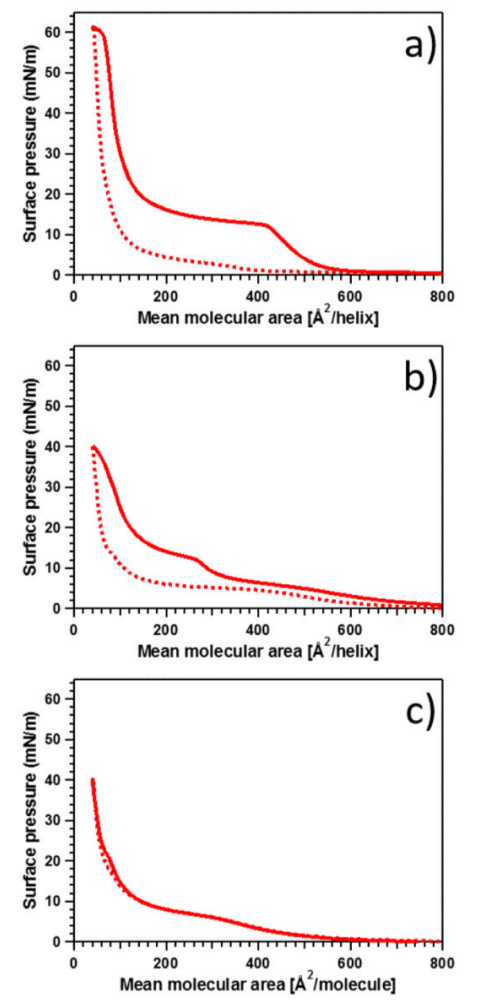

Figure 1.

Isotherms of amphiphilic peptide-polymer conjugates at $25^{\circ} \mathrm{C}$ : (a) $\mathrm{dC} 16-1 \mathrm{CW}$, (b) $\mathrm{dC} 16$ 1CW(PEG2k), and (c) dC16-SC(PEG2k). The solid and dotted lines represent compression and expansion, respectively. No significant hysteresis was found in the case of the scrambled amphiphile. 

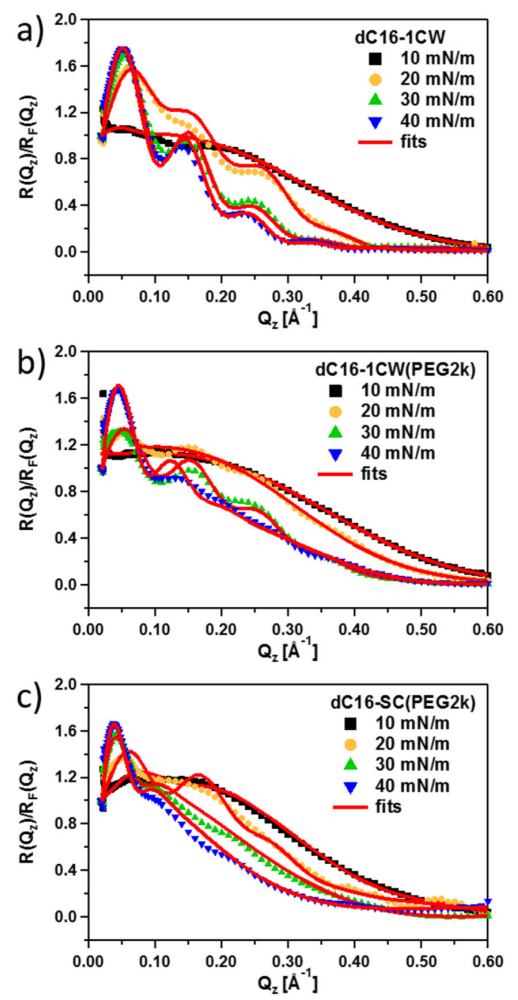

Figure 2.

Fresnel-normalized XR data of the various amphiphilic peptide-polymer conjugates: (a) dC16-1CW, (b) dC16-1CW-(PEG2k), and (c) dC16-SC(PEG2k). The lines show the best fits based on the model described in the text. 

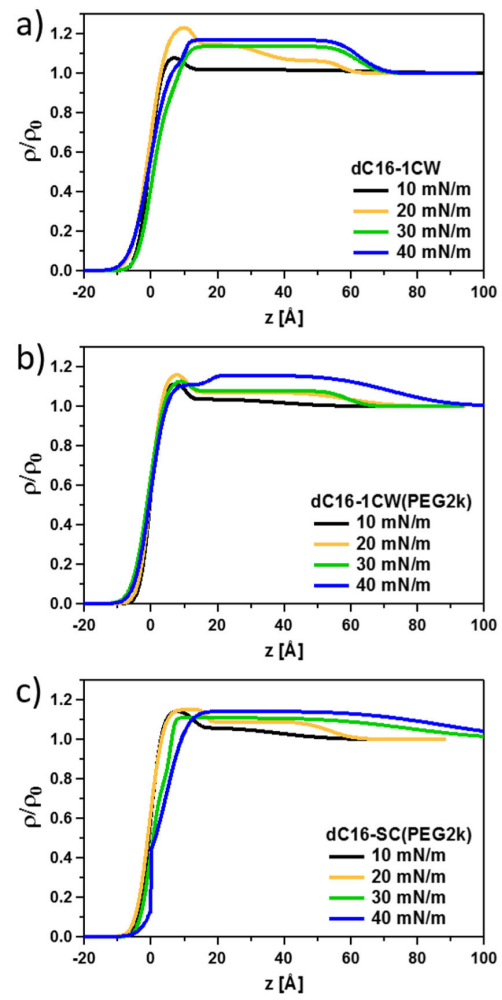

Figure 3.

Density profiles along the air-water interface obtained by model fits of the data displayed in Figure 2 for (a) dC16-1CW, (b) dC16-1CW(PEG2k), and (c) dC16-SC(PEG2k). 


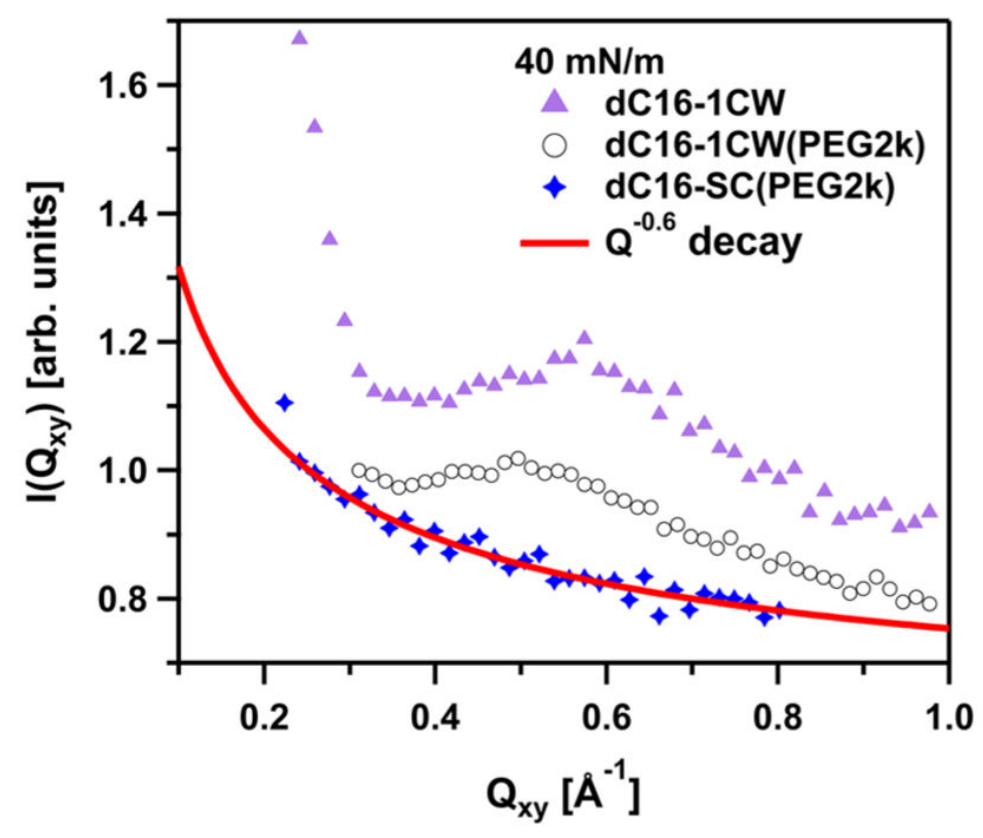

Figure 4.

Integrated GIXD data showing the $Q_{x y}$-dependence of the in plane scattering of the three amphiphiles at a surface pressure of $\Pi=40 \mathrm{mN} / \mathrm{m}$. The line displays a fit to a power-law reflecting the diffuse scattering characterizing the capillary wave fluctuations. Note that a predominant peak is visible for the $\boldsymbol{a}$-helical forming peptide-conjugates but not for scrambled dC16-SC(PEG2k). 

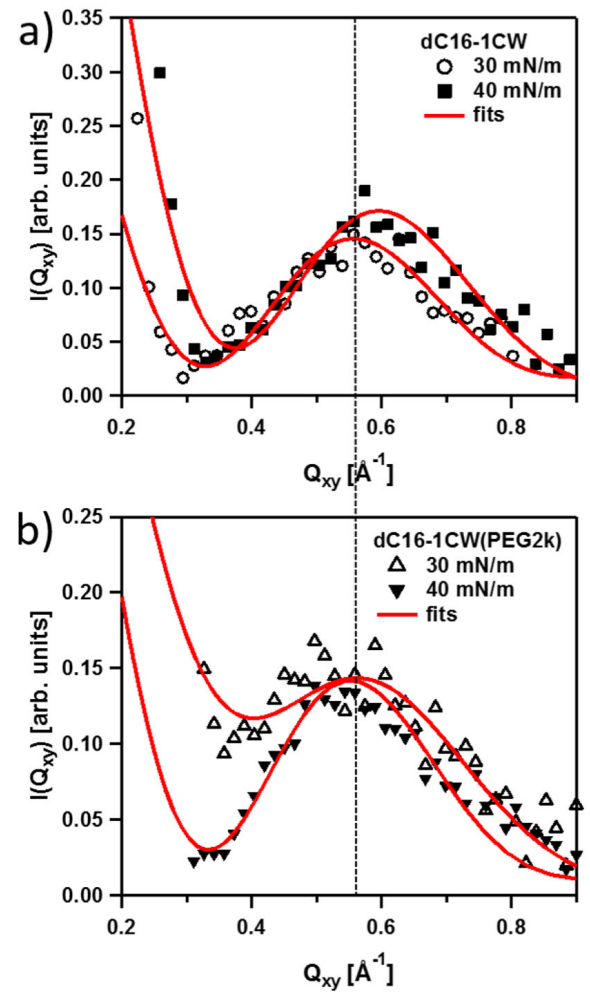

Figure 5.

GIXD data showing the $Q_{x y}$-dependence of the in plane scattering of (a) dC16-1CW and (b) dC16-1CW(PEG2k) at surface pressures of 30 and $40 \mathrm{mN} / \mathrm{m}$. The solid lines represent fits using the bundle model described in the text. 

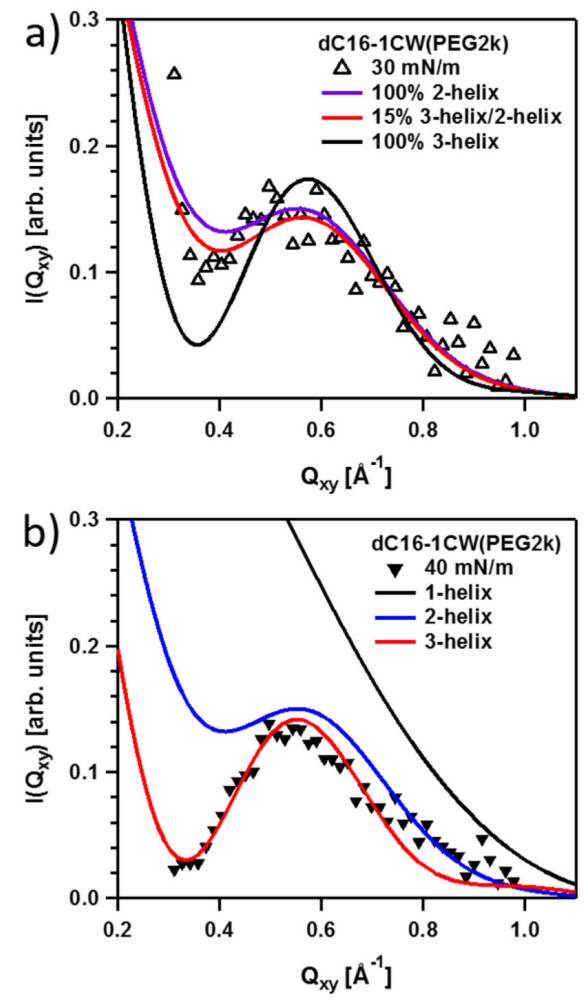

Figure 6.

Comparison of fit results using 2-helix/3-helix bundles and mixtures of the $\mathrm{dC} 16-$ $1 \mathrm{CW}(\mathrm{PEG} 2 \mathrm{k}$ ) data at (a) 30 and (b) $40 \mathrm{mN} / \mathrm{m}$. 

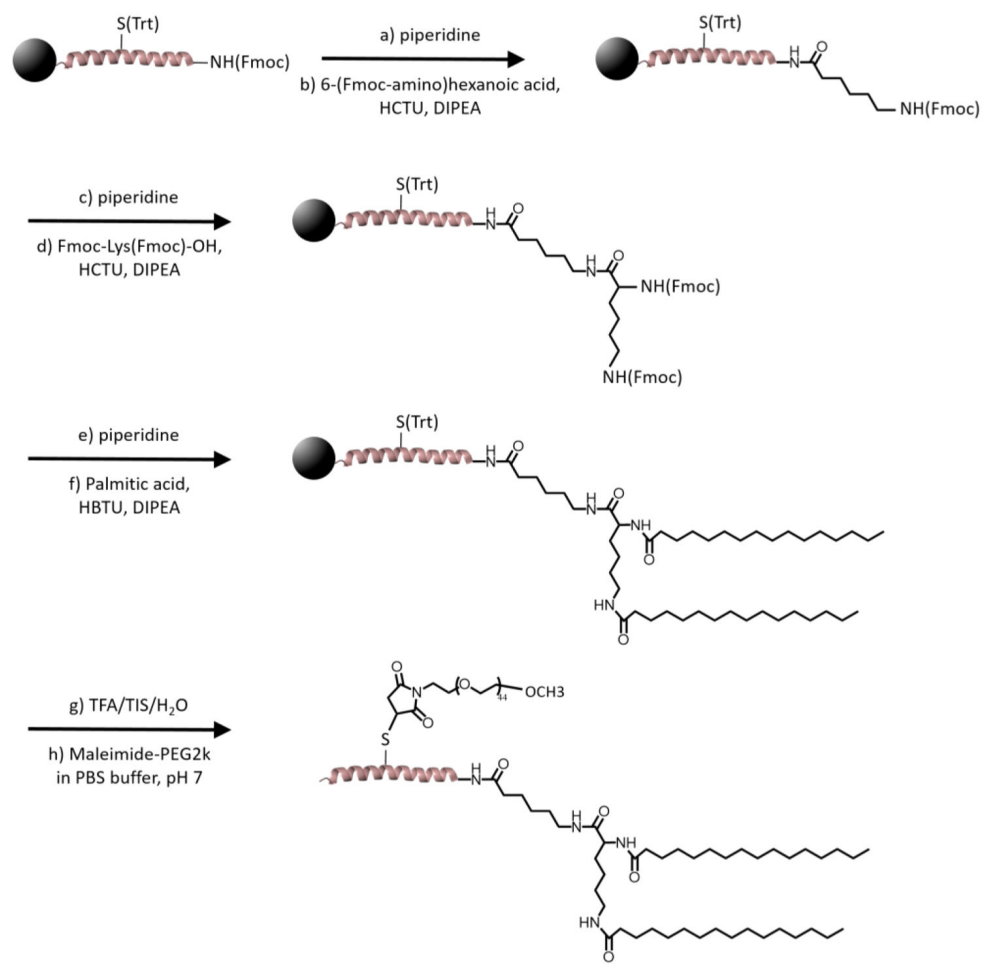

\section{Scheme 1.}

Synthetic Scheme of Amphiphilic Peptide-Polymer Conjugates ${ }^{a}$

${ }^{a}$ For PEGylated conjugates (dC16-1CW(PEG2k) and dC16-SC(PEG2k)), S14C mutation was used to facilitate site-specific coupling of PEG2k. For non-PEGylated dC16-1CW, residue 14 of the peptide sequence remained as serine and step (h) of the synthetic scheme was not performed. 